\title{
SOFT TISSUE SARCOMAS - SERIES OF CASES IN ONE SURGICAL DEPARTMENT
}

doi: 10.2478/rojost-2018-0061

\author{
R.V. Costea, N. Zărnescu, A. Chirca, O. Rusu, S. Neagu \\ "Carol Davila" University of Medicine and Pharmacy, Bucharest, Romania; \\ 2nd Department of Surgery, University Emergency Hospital, Bucharest, Romania
}

Introduction. Soft tissue sarcomas (STS) are a heterogeneous group of tumors with over 80 different subtypes that account for approximately 1-2\% of adult malignancies. Primary sarcomas arise from a variety of soft tissues and bone, and include fibrous connective, fat, and smooth, or striated muscle, vascular, peripheral neural and visceral tissue. With difficulties in establishing cell origin and pathogenesis, this condition lacks an effective and durable therapy with no predictive biomarkers and rapid diagnosis.

Materials and methods. We reviewed the cases of 21 patients treated in our clinic for soft tissue sarcoma over a 20-year period (1999-2018). We extracted the following information from each patient's medical record: disease status at presentation, histological diagnosis, American Joint Committee on Cancer staging, surgical procedure, oncological outcome, length of hospitalization, and follow-up information.

Results. All 21 patients (14 males and 7 females, aged between 19 and 88 years) underwent surgery and total excision with safety margins was performed with histopathological confirmation stating the tumor type and subtype. Some samples required immunohistochemistry for subtype differentiation. Chondrosarcoma and myosarcoma were the most common (5 patients). 8 patients presented local recurrence and metastatic disease with 6 cases receiving adjuvant chemotherapy. Only one patient presented for the 5 -year follow-up.

Conclusions. STS are a rare group of tumors with poor outcome with surgical treatment being represented by total excision. We chose to present our clinic's experience to highlight the need for post-operative therapy advancements and to raise awareness on the difficulties in managing these cases.

Keywords: soft tissue sarcomas, surgical excision, post-operative treatment 